\title{
CXCR4 Blockade Attenuates Hyperoxia-Induced Lung Injury in Neonatal Rats
}

\author{
Shelley Drummond ${ }^{a, b}$ Shalini Ramachandran ${ }^{a, b}$ Eneida Torres ${ }^{a, b}$ \\ Jian Huang ${ }^{a, b}$ Dorothy Hehre ${ }^{a, b}$ Cleide Suguihara ${ }^{a, b} \quad K^{2}$ Karen C. Young ${ }^{a-c}$ \\ ${ }^{a}$ Division of Neonatology, Department of Pediatrics, ${ }^{\mathrm{b}}$ Batchelor Children's Research Institute, and \\ 'The Interdisciplinary Stem Cell Institute, University of Miami Miller School of Medicine, Miami, Fla., USA
}

\section{Key Words}

CXCR4 blockade · Bronchopulmonary dysplasia .

Angiogenesis · Hyperoxia

\begin{abstract}
Background: Lung inflammation is a key factor in the pathogenesis of bronchopulmonary dysplasia (BPD). Stromal-derived factor-1 (SDF-1) and its receptor chemokine receptor 4 (CXCR4) modulate the inflammatory response. It is not known if antagonism of CXCR4 alleviates lung inflammation in neonatal hyperoxia-induced lung injury. Objective: We aimed to determine whether CXCR4 antagonism would attenuate lung injury in rodents with experimental BPD by decreasing pulmonary inflammation. Methods: Newborn rats exposed to normoxia (room air, $\mathrm{RA}$ ) or hyperoxia $\left(\mathrm{FiO}_{2}=0.9\right)$ from postnatal day $2(P 2)$ to $P 16$ were randomized to receive the CXCR4 antagonist, AMD3100 or placebo (PL) from P5 to $P 15$. Lung alveolarization, angiogenesis and inflammation were evaluated at P16. Results: Compared to the RA pups, hyperoxic PL pups had a decrease in alveolarization, reduced lung vascular density and increased lung inflammation. In contrast, AMD3100-treated hyperoxic pups had improved alveolarization and increased angiogenesis. This improvement in lung structure was accompanied by a decrease in
\end{abstract}

the macrophage and neutrophil counts in the bronchoalveolar lavage fluid and reduced lung myeloperoxidase activity. Conclusion: CXCR4 antagonism decreases lung inflammation and improves alveolar and vascular structure in neonatal rats with experimental BPD. These findings suggest a novel therapeutic strategy to alleviate lung injury in preterm infants with BPD.

(c) 2015 S. Karger AG, Basel

\section{Background}

Bronchopulmonary dysplasia (BPD) is characterized by an arrest of alveolar and vascular development [1]. Inflammation plays a major role in the pathogenesis of BPD [2]. This inflammatory response is believed to be triggered antenatally by intrauterine infection and augmented postnatally by factors such as hyperoxia and systemic infections [2]. Preterm infants at various stages in the development of BPD have increased numbers of inflammatory cells in their tracheal aspirate [3]. These inflammatory cells, recruited to the lung in the earliest phase of lung injury, initiate a cascade of injurious events which increase pulmonary microvascular edema and suppress lung growth.

\section{KARGER 125}

(c) 2015 S. Karger AG, Base

$1661-7800 / 15 / 1074-0304 \$ 39.50 / 0$

E-Mail karger@karger.com

www.karger.com/neo
Karen C. Young, MD

Batchelor Children's Research Institute

University of Miami Miller School of Medicine

1580 NW 10th Avenue RM-345, Miami, FL 33136 (USA)

E-Mail KYoung3@med.miami.edu 
Chemokines are peptides which orchestrate the migration of cells involved in inflammatory responses. Initially cloned from bone marrow stromal cells in 1993, the chemokine, stromal-derived factor-1 (SDF-1), is secreted by several tissues, with its major cellular sources being bone marrow stromal cells, macrophages, neutrophils, vascular endothelial cells and fibroblasts [4]. Its cognate receptor, CXCR4, is a G-protein-coupled receptor that is widely expressed on several tissues, including endothelial cells, fibroblasts, neutrophils, monocytes, hematopoietic and tissue-committed stem cells [5]. Although the role of CXCR4/SDF-1 in BPD pathogenesis is unclear, Deng et al. [6] demonstrated increased CXCR4-positive bone marrow-derived fibroblasts in the lungs of rodents exposed to hyperoxia, and these cells appeared to migrate to the lung under the direction of SDF-1.

CXCR4 blockade is a strategy to reduce lung inflammation and repair the injured lung. AMD3100 is a symmetric bicyclam potent nonpeptide CXCR4 antagonist [7]. This compound was first utilized to block the entry of the HIV virus into cells [7]. Although its current clinical use is restricted to adjunctive cancer therapy, preclinical evidence is accumulating to suggest that CXCR4 blockade with AMD3100 facilitates organ repair by decreasing tissue inflammation and increasing progenitor cell migration to areas of injury [8]. CXCR4 antagonism has been shown to decrease cockroach allergy-induced airway inflammation and bleomycin-induced pulmonary inflammation in rodents $[9,10]$. In addition, a single dose of AMD3100 administered to mice with myocardial infarction was found to reduce fibrosis and inflammatory cell incorporation [8].

This study sought to ascertain whether CXCR4 blockade would attenuate hyperoxia-induced lung injury (HILI) in neonatal rats. We demonstrate that CXCR4 antagonism decreases lung inflammation in neonatal rats with HILI and this is accompanied by an improvement in lung vascular density and alveolarization. These findings suggest that CXCR4 blockade have potential as a strategy to reduce BPD in preterm neonates.

\section{Methods}

\section{Animals}

Pregnant Sprague-Dawley rats were purchased from Charles River Laboratories (Wilmington, Mass., USA) and cared for according to NIH guidelines for the use and care of animals during the experimental protocol. Rats were housed in a temperature-regulated room. Their chambers were cleaned twice weekly, and food and water were replaced as needed.

AMD3100 and Hyperoxia-Induced Lung Injury

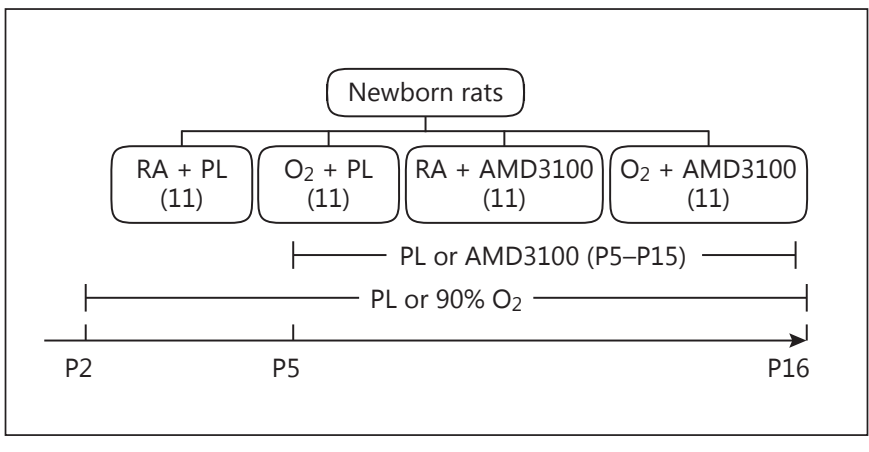

Fig. 1. Experimental design: newborn pups (P2) exposed to RA or hyperoxia $\left(90 \% \mathrm{O}_{2}\right)$ were randomly assigned to receive AMD3100 or PL from P2 to P15. They were evaluated on P16.

\section{Experimental Design}

All animal experiments were performed according to guidelines set forth by the University of Miami Animal Care and Use Committee. At delivery, rat pups ( $\mathrm{n}=44,4$ litters in total) were randomly separated into 4 groups. They were exposed to either normobaric hyperoxia $\left(\mathrm{FiO}_{2}=0.9\right)$ or room air $\left(\mathrm{RA} ; \mathrm{FiO}_{2}=0.21\right)$ from postnatal day 2 (P2) to $\mathrm{P} 16$. The rat mothers were rotated every $48 \mathrm{~h}$ between the hyperoxia and normoxic chambers to prevent oxygen toxicity, and standardized nutrition was provided to each litter. There were no deaths in the RA groups. There was, however, 1 death in each of the hyperoxia groups.

\section{AMD3100 Administration}

Rat pups exposed to hyperoxia or normoxia from P2 to P16 were randomly assigned to receive daily subcutaneous injections of AMD3100 (240 $\mu \mathrm{g} / \mathrm{kg}$; Sigma-Aldrich, Saint Louis, Mo., USA) or vehicle (sterile water) as placebo (PL) from P5 to P15. The dose was chosen based on previous studies showing efficacy with this dose [11]. The animals were studied on P16 (fig. 1).

\section{Assessment of Pulmonary Hypertension}

Right ventricular systolic pressure (RVSP) was measured as a surrogate of pulmonary artery pressure. The weight ratio of right ventricle to left ventricle and septum, i.e. $R V / L V+S$, was utilized as an index of RV hypertrophy.

\section{Assessment of Lung Alveolarization}

Lung morphometric analysis was performed as previously described [12]. Serial paraffin-embedded lung sections $5 \mu \mathrm{m}$ thick, taken from the upper and lower lobes, were stained with standard hematoxylin and eosin (HE). Alveolarization was determined by measuring the mean linear intercept (MLI) and septal density. Images from five randomly selected, nonoverlapping parenchymal fields were acquired from the lung sections of each animal $(n=10$ / group) at $\times 20$ magnification.

\section{Assessment of Vascular Density}

The 5- $\mu \mathrm{m}$-thick, mid-lung sections of the upper and lower lobes were deparaffinized, rehydrated and stained with polyclonal rabbit anti-human von Willebrand factor (vWF; Dako Corp., Carpinteria, Calif., USA). Six randomly selected, nonoverlapping pa- 
renchymal fields were evaluated from the lung sections of each animal (5-6/group). The number of vWF-positive blood vessels/ hpf (20-50 $\mu \mathrm{m}$ in diameter) were counted by a blinded observer.

\section{Assessment of Pulmonary Vascular Remodeling}

Paraffin-embedded lung sections were stained with polyclonal rabbit anti-human vWF and monoclonal mouse anti- $\alpha$-smooth muscle actin (1:500, Sigma-Aldrich). The medial wall thickness (MWT) of partially and fully muscular arteries $(20-50 \mu \mathrm{m}$ in diameter) was determined by using the formula: $2 \mathrm{MT} \times 100 / \mathrm{ED}$, where MT is the distance between the internal and external elastic laminae and ED is the external diameter. Approximately 20 randomly chosen arteries were evaluated per slide and all morphometric analyses were performed by a blinded observer.

\section{Bronchoalveolar Lavage Fluid Analysis}

Bronchoalveolar lavage fluid (BALF) was obtained as previously described [13], and differential cell counts were performed on the cytospin preparations after Giemsa staining.

\section{Western Blot}

The protein expression of matrix metalloproteinase-9 (MMP9), CXCR4 and vascular endothelial growth factor receptor 2 (VEGFR2) in lung homogenates was determined by Western blot analysis. The polyclonal antibodies for CXCR4 (1:500), MMP-9 (1:500) and VEGFR2 (1:200) were obtained from Abcam (Cambridge, Mass., USA) and Cell Signaling Technology (Danvers, Mass., USA), respectively. Lung homogenates were separated by $10 \%$ SDSPAGE, transferred to nitrocellulose membranes and blocked overnight at $4^{\circ} \mathrm{C}$ in $5 \%$ bovine serum albumin. Immunodetection was performed by incubating the membranes with the primary antibodies diluted in blocking buffer for $1 \mathrm{~h}$ at room temperature. After washing, a semiluminescent horseradish peroxidase substrate was diluted in blocking buffer and applied for $60 \mathrm{~min}$. Band intensity was quantified with Quantity One software (Bio-Rad, Hercules, Calif., USA).

\section{Quantitative Real-Time PCR}

RNA from lung tissue was extracted (RNeasy Midi Kit, Qiagen, Inc., Valencia, Calif., USA) and reverse-transcribed. The specific cDNA for IL-6 was quantified by real-time RT-PCR using SuperArray (Frederick, Md., USA) following the real-time gene expression assay protocol. Primers for IL- 6 and GAPDH (as an internal control) were predeveloped by SuperArray. The relative quantity of IL-6 was normalized to GAPDH expression.

\section{VEGF and SDF-1 ELISA}

Lung vascular endothelial growth factor (VEGF-A) and SDF-1 tissue content were quantified using ELISA kits obtained from R\&D Systems (Minneapolis, Minn., USA).

\section{Myeloperoxidase Activity Assay}

Lung myeloperoxidase (MPO) activity was determined using a specific MPO colorimetric activity assay kit as per manufacturer specifications (Biovision, Mountainview, Calif., USA).

\section{Assessment of Lung Fibrosis}

Lung sections were stained with Masson's trichrome stain. Lung collagen content was determined by performing a Sircol collagen assay as per manufacturer specifications (Biocolor, Carrickfergus, Northern Ireland).

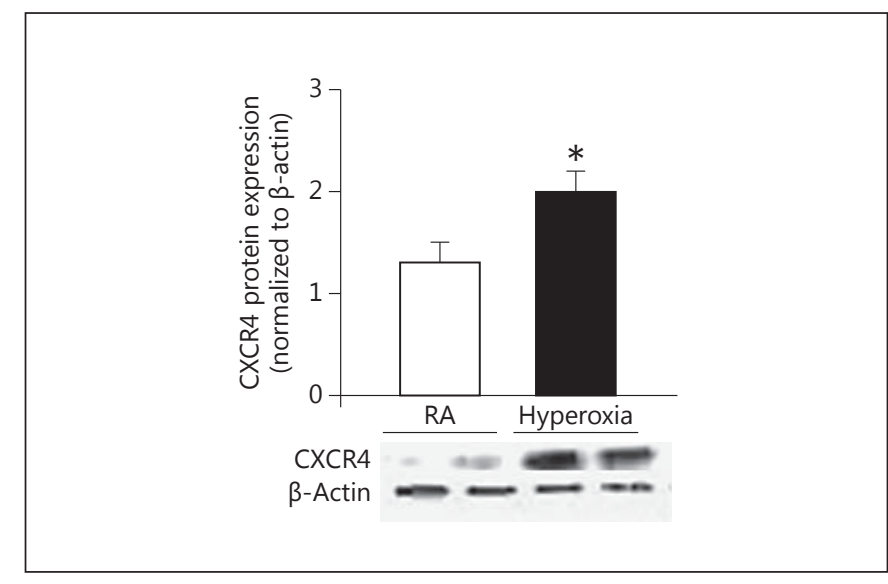

Fig. 2. Increased lung CXCR4 expression in HILI. Increased lung CXCR4 protein expression in newborn rats exposed to 14 days of hyperoxia. ${ }^{*} \mathrm{p}<0.002$, RA vs. hyperoxia ( $\mathrm{n}=5$ /group). CXCR4 expression is normalized to $\beta$-actin. A representative Western blot is shown in the lower panel.

\section{Statistics}

Results are reported as mean \pm SD. Data were analyzed by twoway ANOVA followed by a post hoc analysis (Holm-Sidak). Values of $\mathrm{p}<0.05$ were considered statistically significant.

\section{Results}

\section{Lung CXCR4 Expression Is Increased in Neonatal HILI}

We first sought to ascertain whether hyperoxia exposure would affect the protein expression of CXCR 4 in the lungs of neonatal pups. Whole-lung lysates were obtained from newborn rat pups exposed to normoxia or hyperoxia $\left(90 \% \mathrm{O}_{2}\right)$ for 14 days. The protein expression of CXCR4 was determined by Western blot. Compared to normoxic pups, there was an approximately 2 -fold increase ( $\mathrm{p}<0.002 ; \mathrm{n}=5$ /group) in the protein expression of CXCR4 in the lung lysates obtained from the hyperoxic pups (fig. 2). There was, however, no change in the lung tissue content of SDF- $1(0.396 \pm 0.06$ vs. $0.459 \pm 0.06$ $\mathrm{ng} / \mathrm{ml}$, normoxia vs. hyperoxia, $\mathrm{p}=0.07, \mathrm{n}=5$ /group) following 14 days of hyperoxia.

\section{CXCR4 Blockade Improves Alveolarization in Neonatal HILI}

There was no difference in the degree of alveolarization between the RA groups (fig. 3a). Hyperoxia-exposed animals showed a marked simplification of the alveoli, in the form of larger alveoli with increased alveolar diame- 

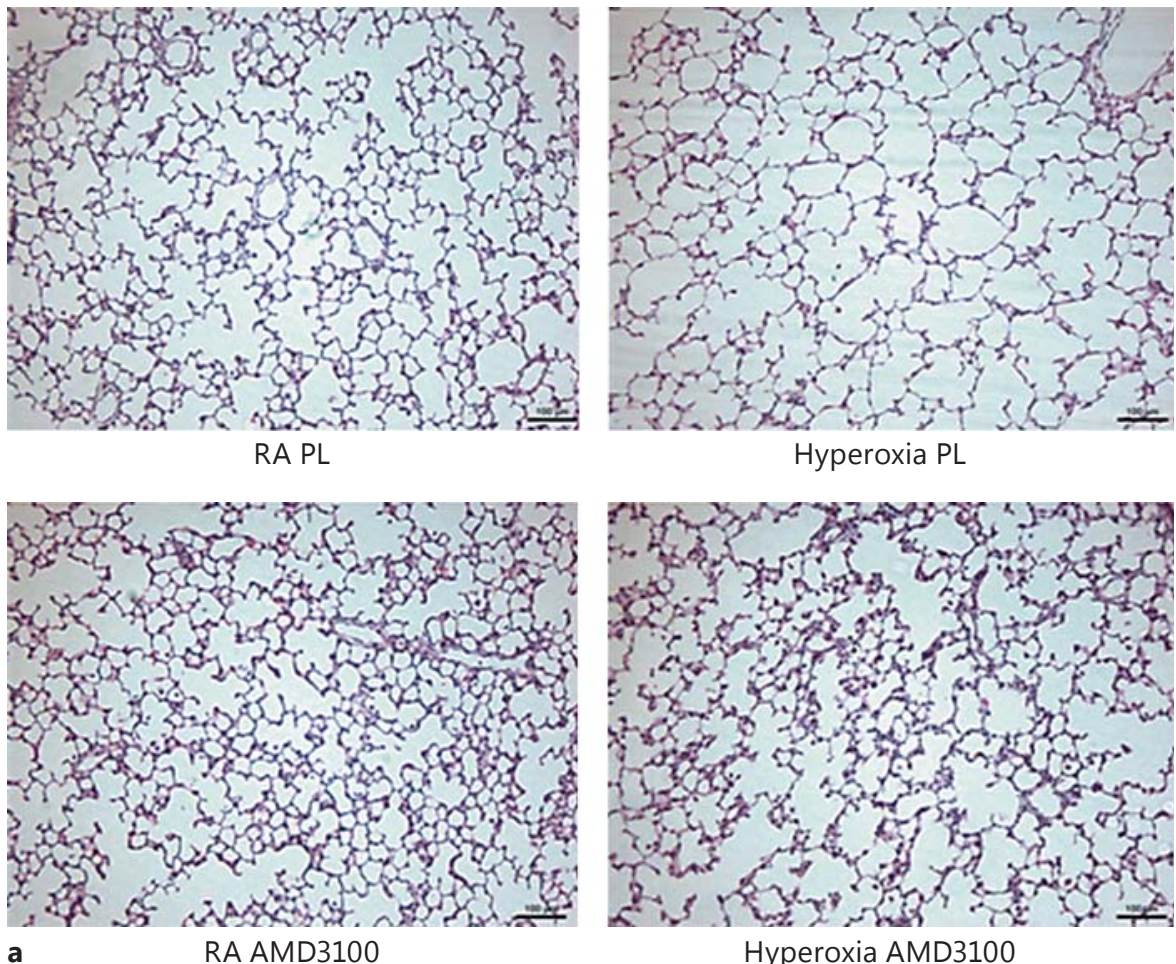

Hyperoxia PL

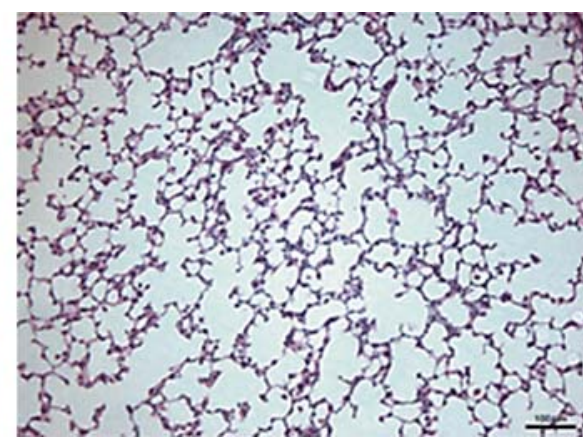

Hyperoxia AMD3100

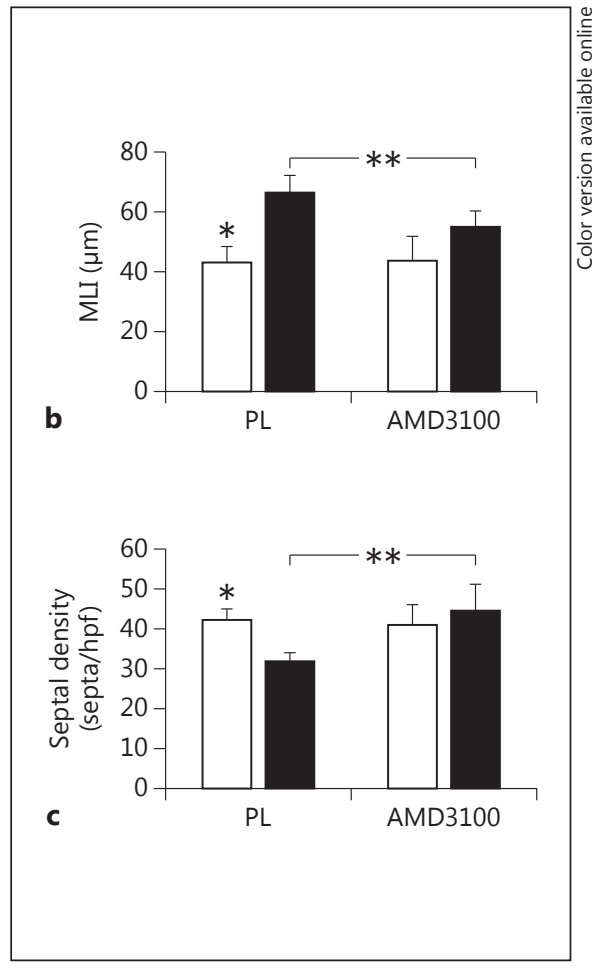

PL vs. hyperoxia AMD3100 ( $\mathrm{n}=10$ /group). White bars represent RA animals and black bars represent hyperoxia animals. c Increased septal density in hyperoxia AMD3100-treated rats. ${ }^{*} \mathrm{p}<$ 0.0001 , RA PL vs. hyperoxia PL; ${ }^{* *} \mathrm{p}<0.002$, hyperoxia PL vs. hyperoxia AMD3100 ( $\mathrm{n}=10$ /group). ters and decreased septation. Furthermore, compared to RA animals, there was an increase in MLI ( $43 \pm 5$ vs. $66 \pm$ $5 \mu \mathrm{m}, \mathrm{RA}$ PL vs. hyperoxia PL, $\mathrm{p}<0.05, \mathrm{n}=10$ /group), and a decrease in alveolar septation in the hyperoxia-exposed animals ( $42 \pm 3$ vs. $32 \pm 2$ septa/hpf, RA PL vs. hyperoxia PL, $\mathrm{p}<0.0001, \mathrm{n}=10$ /group; fig. $3 \mathrm{~b}, \mathrm{c}$ ). In contrast, the administration of AMD3100 significantly improved alveolarization, as shown by increased secondary septation ( $32 \pm 2$ vs. $45 \pm 6$ septa/hpf, hyperoxia PL vs. hyperoxia AMD3100, $\mathrm{p}<0.002, \mathrm{n}=10$ /group) and decreased MLI ( $66 \pm 5$ vs. $55 \pm 5 \mu \mathrm{m}$, hyperoxia PL vs. hyperoxia AMD3100, $\mathrm{p}<0.007, \mathrm{n}=10$ /group).

\section{CXCR4 Blockade Increases Vascular Density in \\ Hyperoxia-Induced Lung Injury}

Compared to the RA animals, the hyperoxia-exposed pups demonstrated decreased vascular density (fig. 4a, b). In contrast, the administration of AMD3100 to hyperoxic rats increased the lung vascular density by approximately 2 -fold. These findings were associated with an in- crease in lung VEGF protein concentration ( $870 \pm 18$ vs. $1,420 \pm 61 \mathrm{pg} / \mathrm{ml}$, hyperoxia PL vs. hyperoxia AMD3100, $\mathrm{p}<0.0001, \mathrm{n}=5$ /group) and VEGFR2 protein expression (hyperoxia PL vs. hyperoxia AMD3100, $\mathrm{p}<0.02, \mathrm{n}=5$ / group; fig. $4 c, d$ ). There was no difference between the hyperoxic groups in the RVSP, RV/LV $+\mathrm{S}$ or the degree of pulmonary vascular remodeling (i.e. MWT; fig. $4 \mathrm{e}-\mathrm{g}$ ).

\section{CXCR4 Blockade Decreases Inflammation in HILI}

The hyperoxia-exposed rats showed increased numbers of BALF macrophages and neutrophils, respectively, compared to the rats exposed to RA $\left(4 \times 10^{4} \pm 1 \times 10^{3} \mathrm{vs}\right.$. $32 \times 10^{4} \pm 14 \times 10^{4}$ cells $/ \mathrm{ml}$, RA PL vs. hyperoxia PL, $\mathrm{p}<$ $0.0001, \mathrm{n}=5$ /group and $0.8 \times 10^{4} \pm 0.2 \times 10^{3}$ vs. $3.5 \times 10^{4}$ $\pm 2 \times 10^{4}$ cells $/ \mathrm{ml}$, RA PL vs. hyperoxia PL, $\mathrm{p}<0.0001$, $\mathrm{n}=5$ /group; fig. $5 \mathrm{a}, \mathrm{b})$. In contrast, the hyperoxia-exposed, AMD3100-treated rats had markedly decreased BALF macrophage and neutrophil counts to near normoxic levels $\left(32 \times 10^{4} \pm 14 \times 10^{4}\right.$ vs. $5 \times 10^{4} \pm 1 \times 10^{4}$ cells/ $\mathrm{ml}$, hyperoxia PL vs. hyperoxia AMD3100, $\mathrm{p}<0.0001$, 
Fig. 4. CXCR4 blockade increases lung vascular density in HILI. a Lung sections demonstrating improved capillary density in hyperoxia-exposed rats treated with AMD3100. vWF, red and 6-diamidino-2-phenylindole (DAPI; blue; colors refer to the online version only). $\times 100$. b Increased vascular density in hyperoxia AMD3100 rats. ${ }^{*} \mathrm{p}<0.0001$, RA PL vs. hyperoxia PL; ${ }^{* *} \mathrm{p}<0.05$, hyperoxia PL vs. hyperoxia AMD3100 ( $n=10$ /group). White bars represent RA animals and black bars represent hyperoxia animals. c Increased lung VEGF concentration in hyperoxia AMD3100 rats. ${ }^{*} \mathrm{p}<0.05$, RA PL vs. hyperoxia PL; ${ }^{* *} \mathrm{p}<0.0001$, hyperoxia $\mathrm{PL}$ vs. hyperoxia AMD3100 ( $\mathrm{n}=5$ /group). d Increased lung VEGFR2 expression in hyperoxia AMD3100 rats. ${ }^{*} \mathrm{p}<0.004, \mathrm{RA}$ $\mathrm{PL}$ vs. hyperoxia $\mathrm{PL} ;{ }^{* *} \mathrm{p}<0.02$, hyperoxia PL vs. hyperoxia AMD3100 ( $n=5$ /group). HYP = Hyperoxia. VEGFR2 expression is normalized to $\beta$-actin. e Increased RVSP in hyperoxia groups. ${ }^{*} \mathrm{p}<$ 0.05 , RA PL/AMD3100 vs. hyperoxia PL/AMD3100 ( $\mathrm{n}=10$ /group). There was no difference in the RVSP between the hyperoxia groups. f Increased RV/LV $+\mathrm{S}$ in the hyperoxia groups. ${ }^{*} \mathrm{p}<0.05$, RA PL/ AMD3100 vs. hyperoxia PL/AMD3100 ( $n=10$ /group). There was no difference in the $\mathrm{RV} / \mathrm{LV}+\mathrm{S}$ between the hyperoxia groups. g Increased MWT in the hyperoxia groups. ${ }^{*} \mathrm{p}<0.05$, RA PL/ AMD3100 vs. hyperoxia PL/AMD3100 ( $\mathrm{n}=10$ /group). There was no difference in the MWT between the hyperoxia groups.

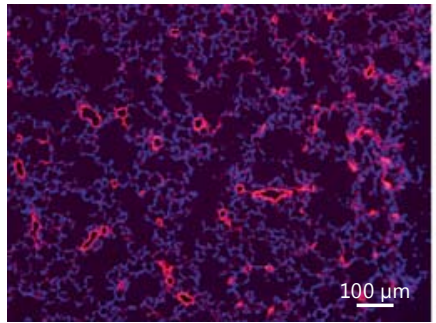

RA PL

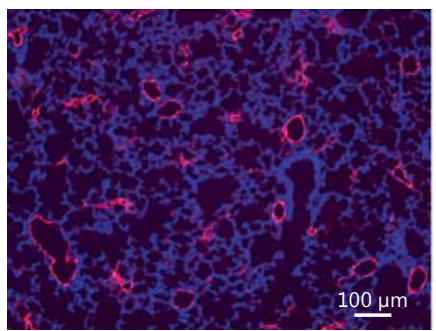

a $\quad$ RA AMD3100

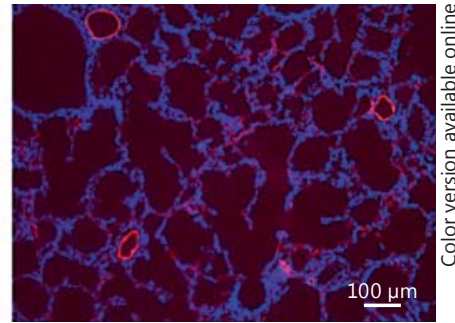

Hyperoxia PL

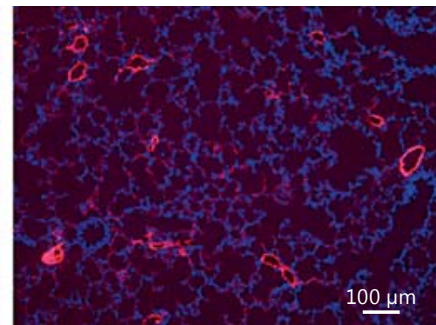

Hyperoxia AMD3100
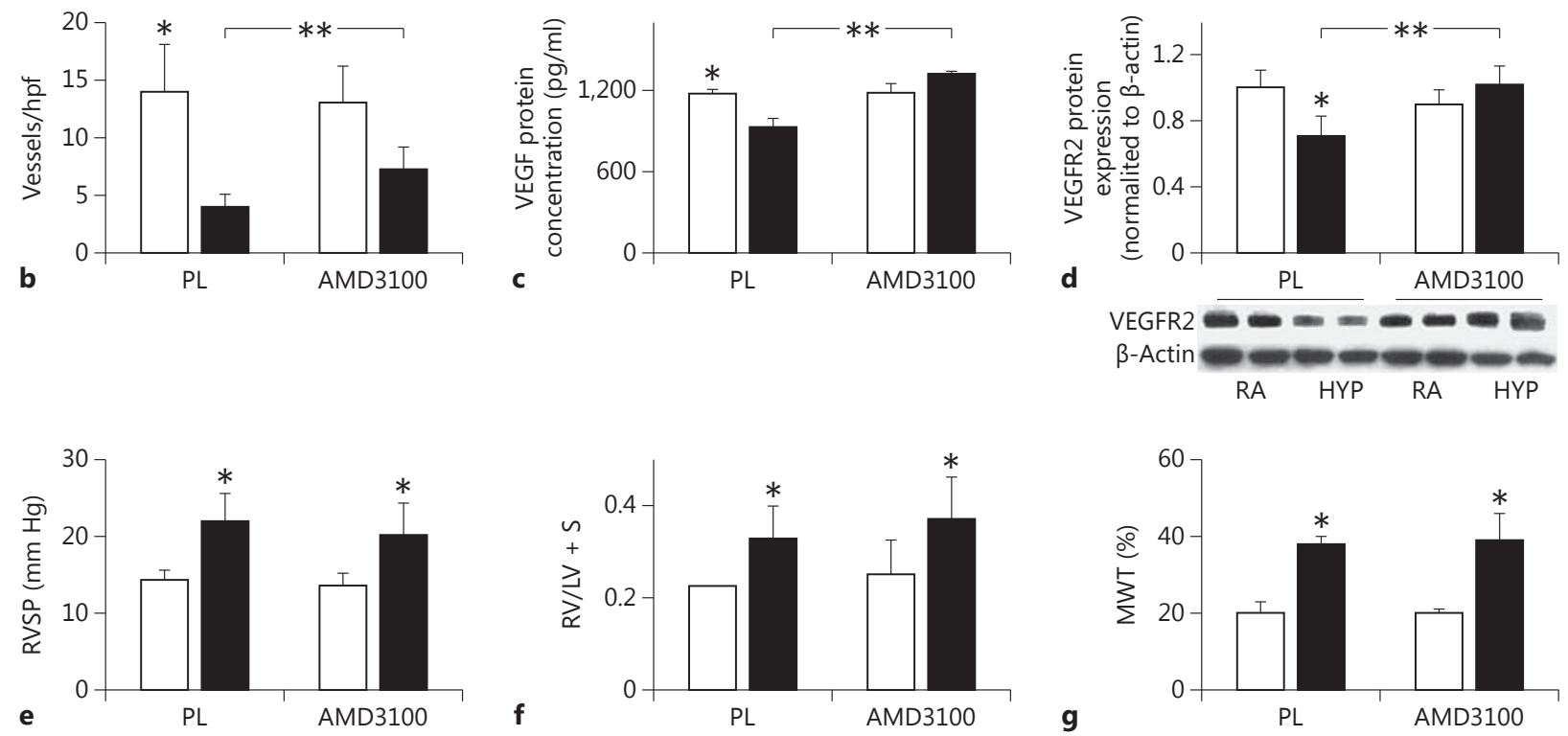

$\mathrm{n}=5$ /group and $3.5 \times 10^{4} \pm 2 \times 10^{4}$ vs. $0.7 \times 10^{4} \pm 0.6 \times$ $10^{4}$ cells/ml, hyperoxia PL vs. hyperoxia AMD3100, $\mathrm{p}<$ $0.0001, \mathrm{n}=5$ /group). These findings were associated with a decrease in lung MPO activity $(0.4 \pm 0.17$ vs. $0.01 \pm$ $0 \mathrm{mU} / \mathrm{ml}$, hyperoxia PL vs. hyperoxia AMD3100, $\mathrm{p}<$ $0.02, \mathrm{n}=5$ /group), MMP-9 expression (4-fold, hyperoxia PL vs. hyperoxia AMD3100, $\mathrm{p}<0.0001, \mathrm{n}=5$ /group; fig. 5c, d) and IL-6 gene expression (50-fold, hyperoxia PL vs. hyperoxia AMD3100, $\mathrm{p}<0.0001, \mathrm{n}=5$ /group).

\section{CXCR4 Blockade Decreases Lung Fibrosis in HILI}

In order to determine the effects of AMD3100 on lung fibrosis, the Masson's trichrome-stained lung sections were evaluated. Compared to the RA rats, the hyperoxia- 

mation in HILI. a Reduced BALF macrophage counts in hyperoxia AMD3100 rats. * $\mathrm{p}<0.0001$, RA PL vs. hyperoxia PL; ** $\mathrm{p}<0.0001$, hyperoxia PL vs. hyperoxia AMD3100 ( $n=5 /$ group). White bars represent RA animals and black bars represent hyperoxia animals. b Decreased BALF neutrophil counts in hyperoxia AMD3100 rats. ${ }^{*} \mathrm{p}<0.0001$, RA PL vs. hyperoxia PL; ** $\mathrm{p}<0.0001$, hyperoxia PL vs. hyperoxia AMD3100 ( $=5 /$ group). c Reduced lung MPO activity in hyperoxia AMD3100 rats. * $\mathrm{p}<0.0001$, RA PL vs. hyperoxia PL; ** $\mathrm{p}<0.02$, hyperoxia PL vs. hyperoxia AMD3100 ( $n=5 /$ group). d Decreased lung MMP-9 protein expression in hyperoxia AMD3100 rats. ${ }^{*} \mathrm{p}<0.05$, RA PL vs. hyperoxia PL; ${ }^{* *} \mathrm{p}<0.05$, hyperoxia PL vs. hyperoxia AMD3100 ( $\mathrm{n}=5$ /group). HYP = Hyperoxia. MMP-9 expression is normalized to $\beta$-actin.
Fig. 5. CXCR4 blockade decreases inflam-
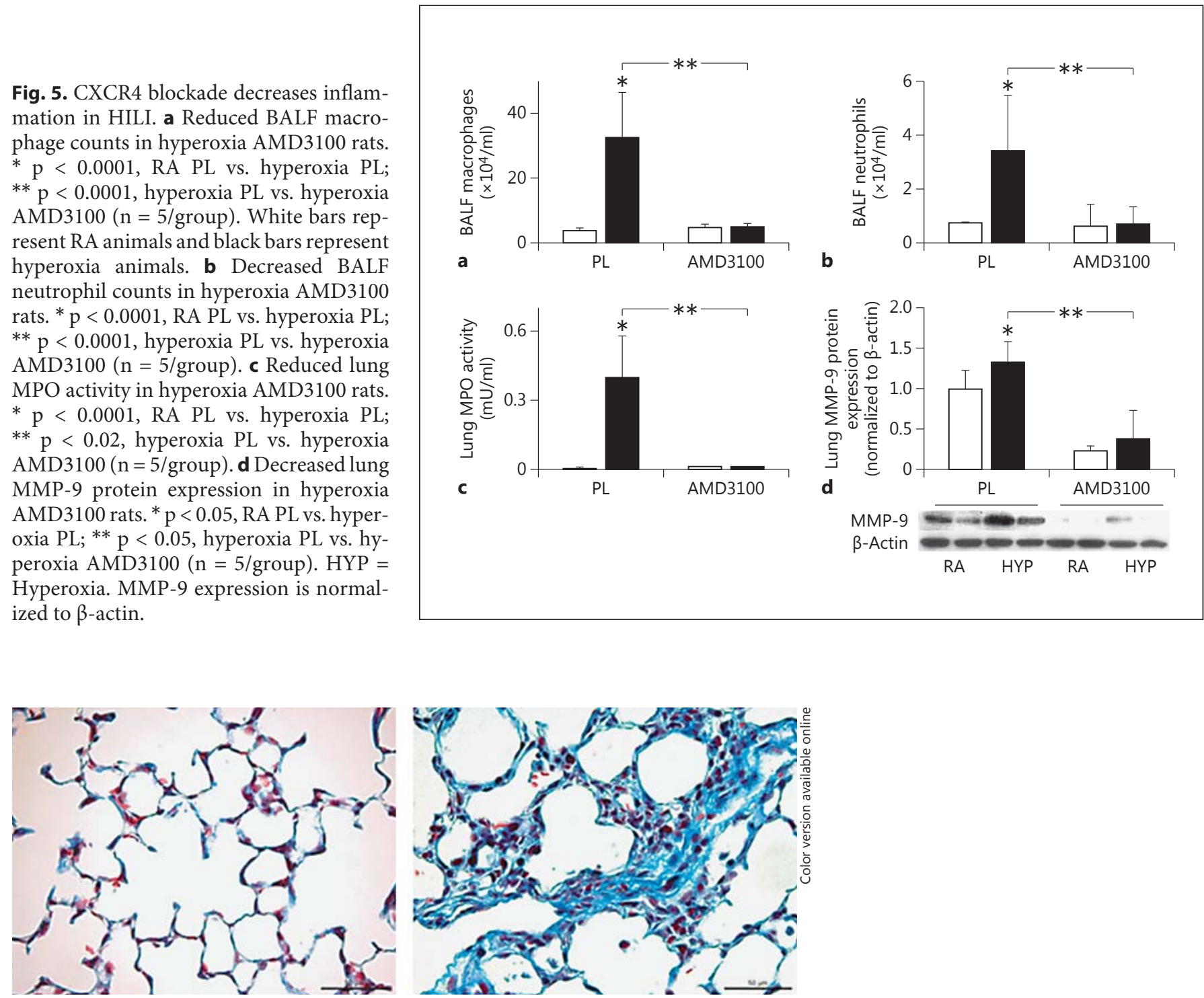

RA PL

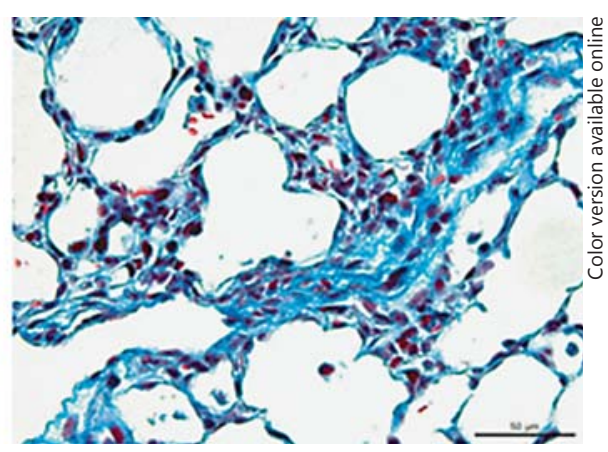

Hyperoxia PL

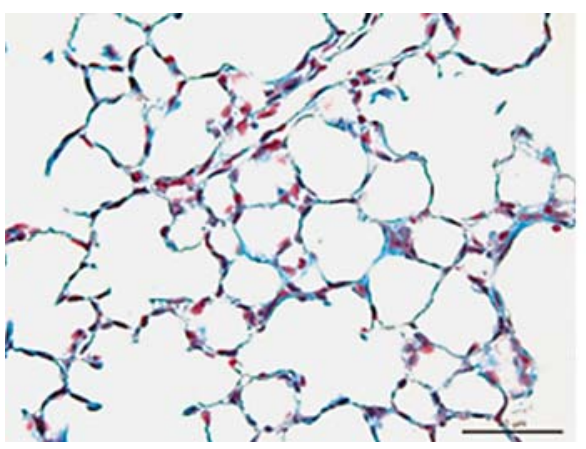

RA AMD3100

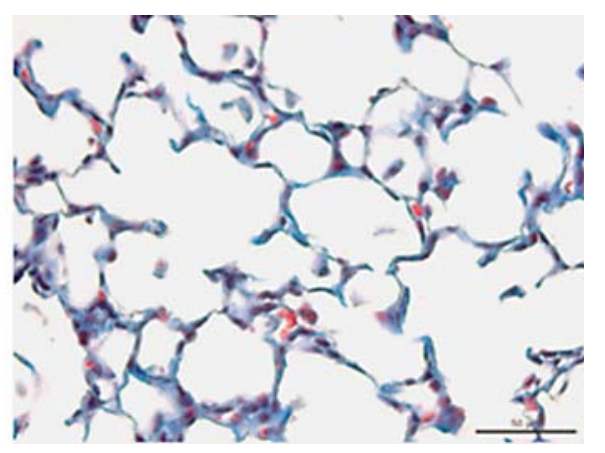

Hyperoxia AMD3100

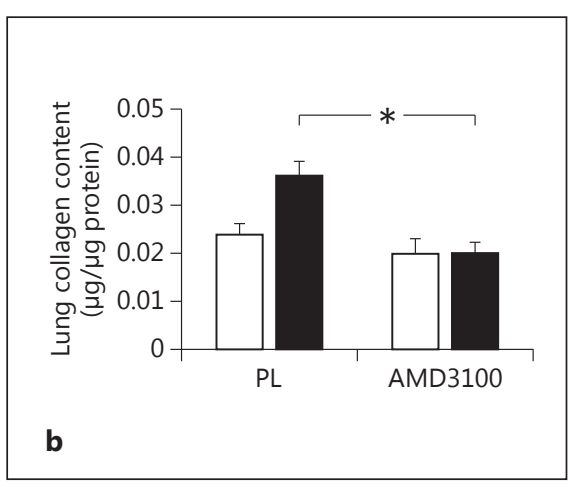

Fig. 6. CXCR4 blockade decreases lung fibrosis. a Lung sections showing decreased lung fibrosis in hyperoxia AMD3100-treated rats. Masson's trichrome. $\times 400$. Scale bars: $50 \mu \mathrm{m}$. b Decreased lung collagen in hyperoxia AMD3100-treated rats. ${ }^{*} \mathrm{p}<0.0001$,
RA PL vs. hyperoxia PL and hyperoxia PL vs. hyperoxia AMD3100 ( $n=5$ /group). White bars represent RA animals and black bars represent hyperoxia animals. 
exposed rats had lung fibrosis and increased lung collagen (fig. 6a, b). In contrast, the administration of AMD3100 to hyperoxic rats decreased lung fibrosis and collagen content. There was no difference in lung collagen content between the RA PL and hyperoxia AMD3100 groups.

\section{Discussion}

This study sought to ascertain whether CXCR4 blockade would attenuate neonatal hyperoxia-induced lung injury, an experimental model of BPD. We show that the administration of the CXCR4 antagonist AMD3100 to neonatal rodents with experimental BPD decreases lung inflammation, improves alveolarization and angiogenesis. Our findings suggest that strategies based on modulating the activity of the SDF-1/CXCR4 axis may be potentially efficacious in repairing the injured preterm lung.

We first demonstrate an increase in lung CXCR4 expression during hyperoxia. This finding is in keeping with those by other several investigators on hyperoxia and LPSinduced lung injury $[6,14]$. Surprisingly, in our study, hyperoxia did not increase the lung SDF-1 tissue content. While our findings are similar to those of Balasubramaniam et al. [15], other investigators have found an increase in lung SDF-1 concentration during hyperoxia [6]. It is possible that the disparity between our findings and those of other investigators is secondary to differences in our animal model. Nonetheless, in agreement with other studies, the absence of an SDF-1 gradient did not affect the anti-inflammatory effects of AMD3100 [16].

In our study, the administration of AMD3100 to hyperoxic pups reduced lung inflammation, shown by the reduction in BALF inflammatory cells and lung MPO activity. Previous studies have shown that inflammation is a key component in the pathogenesis of BPD [2]. Moreover, preterm infants in whom BPD develops have elevated protein levels of inflammatory cytokines and increased numbers of inflammatory cells in their tracheal aspirates [17]. Hyperoxia is one of the most potent inducers of inflammation in these patients. Our finding that CXCR4 blockade reduces lung inflammation in a hyperoxic model of BPD is consistent with those of other investigators who showed that antagonism of the SDF-1/CXCR4 axis reduced lung neutrophil infiltration during LPS-induced lung injury [14]. It is also possible that AMD3100 may have decreased the inflammation by increasing the egress of neutrophils from the lung [18], decreasing inflammatory cell transendothelial migration or by having negative functional effects on other chemokine receptors [19].
The improvement in lung inflammation that we observed was associated with decreased MMP-9 expression. MMP-9 is expressed by several cells, including neutrophils, and it works synergistically with SDF-1 to regulate the transendothelial migration of inflammatory cells [20]. We speculate that the decreased MMP-9 expression in the AMD3100-treated pups was not only due to the decrease in inflammatory cells in the hyperoxic group, but this may also have been secondary to the reduced activation of the SDF-1/CXCR4 downstream signaling pathways which modulate MMP-9 expression [21].

AMD3100 also decreased the lung collagen content in the hyperoxic pups. Increased total lung collagen content has been previously shown in the lungs of infants with BPD [22]. Moreover, Deng et al. [6] demonstrated increased CXCR4-positive fibroblasts in the lungs of rodents with hyperoxia-induced lung injury. Our finding that CXCR4 blockade improves lung collagen content following hyperoxia-induced lung injury is consistent with other studies which have shown decreased lung collagen content in rodent models of bleomycin-induced lung fibrosis following the administration of a CXCR4 antagonist [9].

Interestingly, in our study, although there was an improvement in lung vascular density following AMD3100 administration, there were no significant effects on RVSP, RV hypertrophy or vascular remodeling. Our negative findings may be due to the fact that although there was an improvement in lung vascular density in hyperoxic AMD3100 rats, the number of intra-acinar vessels/hpf was still significantly lower than in the RA PL rats. Previous studies showed decreased vascular remodeling in hypoxic rodent models of pulmonary hypertension following AMD3100 administration [23]. It is plausible that this disparity between studies is secondary to the differences in CXCR4 signaling during hypoxia compared to the hyperoxic conditions in our study, the timing of our intervention and the dynamic processes involved in repair.

Finally, there were also several limitations to our study. The hyperoxic rodent model utilized corresponds to the saccular-alveolar stages of the human lung development model and most preterm infants who develop BPD are in the late canalicular to early saccular stages of lung development. In addition, although hyperoxia is a significant contributor to the pathogenesis of BPD, we utilized a relatively high oxygen concentration which mimics severe BPD. Indeed, most preterm infants are not exposed to this degree of postnatal hyperoxia and thus, potentially, the efficacy of our therapy may be altered. Finally, given in vitro data demonstrating that CXCR4 knockdown im- 
peded alveolar epithelial-cell wound-healing, future studies evaluating the long-term effect of CXCR4 blockade on alveolar epithelial-cell homeostasis will need to be performed [24].

Nonetheless, our study shows that CXCR4 antagonism reduces alveolar growth arrest and impaired angiogenesis in neonatal rodents with a hyperoxia-induced BPD-like phenotype. Although further long-term studies will need to be performed to evaluate the effects of SDF1/CXCR4 axis modulation on other developing organs, these findings suggest that this modulation could be a potential strategy for the treatment of BPD.

\section{Acknowledgements}

We wish to thank Dr. Claudia O. Rodrigues for critiquing this manuscript. This work is supported by a National Institute of Health KO8 Award, Florida Biomedical Research Award and Batchelor Research Foundation Award to K.C.Y.

\section{Disclosure Statement}

None of the authors has a financial relationship with a commercial entity that has an interest in the subject of this paper.

\section{References}

1 Philip AG: Bronchopulmonary dysplasia: then and now. Neonatology 2012;102:1-8.

2 Jobe AJ: The new BPD: an arrest of lung development. Pediatr Res 1999;46:641-643.

3 Munshi UK, Niu JO, Siddiq MM, Parton LA: Elevation of interleukin-8 and interleukin-6 precedes the influx of neutrophils in tracheal aspirates from preterm infants who develop bronchopulmonary dysplasia. Ped Pulmonol 1997;24:331-336

4 McGrath KE, Koniski AD, Maltby KM, McGann JK, Palis J: Embryonic expression and function of the chemokine SDF-1 and its receptor, CXCR4. Develop Biol 1999;213:442456.

5 Ratajczak MZ, Majka M, Kucia M, Drukala J, Pietrzkowski Z, Peiper S, Janowska-Wieczorek A: Expression of functional CXCR4 by muscle satellite cells and secretion of SDF-1 by muscle-derived fibroblasts is associated with the presence of both muscle progenitors in bone marrow and hematopoietic stem/progenitor cells in muscles. Stem Cells 2003;21: 363-371.

6 Deng C, Wang J, Zou Y, Zhao Q, Feng J, Fu Z, Guo C: Characterization of fibroblasts recruited from bone marrow-derived precursor in neonatal bronchopulmonary dysplasia mice. J Appl Physiol 2011;111:285-294.

7 Donzella GA, Schols D, Lin SW, Este JA, Nagashima KA, Maddon PJ, Allaway GP, Sakmar TP, Henson G, De Clercq E, Moore JP: AMD3100, a small molecule inhibitor of HIV-1 entry via the CXCR4 co-receptor. Nat Med 1998;4:72-77.

8 Jujo K, Hamada $\mathrm{H}$, Iwakura $\mathrm{A}$, Thorne $\mathrm{T}$, Sekiguchi H, Clarke T, Ito A, Misener S, Tanaka T, Klyachko E, et al: CXCR4 blockade augments bone marrow progenitor cell recruitment to the neovasculature and reduces mortality after myocardial infarction. Proc Natl Acad Sci USA 2010;107:11008-11013.
9 Xu J, Mora A, Shim H, Stecenko A, Brigham KL, Rojas M: Role of the SDF-1/CXCR4 axis in the pathogenesis of lung injury and fibrosis. Am J Respir Cell Mol Biol 2007;37:291-299.

10 Lukacs NW, Berlin A, Schols D, Skerlj RT, Bridger GJ: AMD3100, a CXCR4 antagonist, attenuates allergic lung inflammation and airway hyperreactivity. Am J Pathol 2002;160: 1353-1360.

11 Devine SM, Flomenberg N, Vesole DH, Liesveld J, Weisdorf D, Badel K, Calandra G, DiPersio JF: Rapid mobilization of CD34+ cells following administration of the CXCR4 antagonist AMD3100 to patients with multiple myeloma and non-Hodgkin's lymphoma. J Clin Oncol 2004;22:1095-1102.

12 Thurlbeck WM: Measurement of pulmonary emphysema. Am Rev Respir Dis 1967;95: 752-764.

13 Hummler SC, Rong M, Chen S, Hehre D, Alapati $\mathrm{D}, \mathrm{Wu} \mathrm{S}$ : Targeting glycogen synthase kinase- $3 \beta$ to prevent hyperoxia-induced lung injury in neonatal rats. Am J Resp Cell Mol Biol 2013;48:578-588.

14 Petty JM, Sueblinvong V, Lenox CC, Jones CC, Cosgrove GP, Cool CD, Rai PR, Brown KK, Weiss DJ, Poynter ME, Suratt BT: Pulmonary stromal-derived factor- 1 expression and effect on neutrophil recruitment during acute lung injury. J Immunol 2007;178:8148-8157.

15 Balasubramaniam V, Mervis CF, Maxey AM, Markham NE, Abman SH: Hyperoxia reduces bone marrow, circulating, and lung endothelial progenitor cells in the developing lung: implications for the pathogenesis of bronchopulmonary dysplasia. Am J Physiol Lung Cell Mol Physiol 2007;292:L1073-L1084.

16 Zuk A, Gershenovich M, Ivanova Y, MacFarland RT, Fricker SP, Ledbetter S: CXCR(4)antagonism as a therapeutic approach to prevent acute kidney injury. Am J Physiol Renal Physiol 2014;307:F783-F797.

17 Ogden BE, Murphy SA, Saunders GC, Pathak D, Johnson JD: Neonatal lung neutrophils and elastase/proteinase inhibitor imbalance. Am Rev Respir Dis 1984;130:817-821.
18 Devi S, Wang Y, Chew WK, Lima R, AGonzález N, Mattar CNZ, Chong SZ, Schlitzer A, Bakocevic N, Chew S, et al: Neutrophil mobilization via plerixafor-mediated CXCR4 inhibition arises from lung demargination and blockade of neutrophil homing to the bone marrow. J Exp Med 2013;210:2321-2336.

19 Sohy D, Yano H, de Nadai P, Urizar E, Guillabert A, Javitch JA, Parmentier M, Springael JY: Hetero-oligomerization of CCR2, CCR5, and CXCR4 and the protean effects of 'selective' antagonists. J Biol Chem 2009;284: 31270-31279.

20 Zhang H, Trivedi A, Lee J-U, Lohela M, Lee SM, Fandel TM, Werb Z, Noble-Haeusslein LJ: Matrix metalloproteinase- 9 and stromal cell-derived factor-1 act synergistically to support migration of blood-borne monocytes into the injured spinal cord. J Neuro 2011;31: 15894-15903.

21 Yu X, Huang Y, Collin-Osdoby P, Osdoby P: Stromal cell-derived factor-1 (SDF-1) recruits osteoclast precursors by inducing chemotaxis, matrix metalloproteinase-9 (MMP-9) activity, and collagen transmigration. J Bone Miner Res 2003;18:1404-1418.

22 Cherukupalli K, Larson JE, Rotschild A, Thurlbeck WM: Biochemical, clinical, and morphologic studies on lungs of infants with bronchopulmonary dysplasia. Ped Pulmonol 1996;22:215-229.

23 Young KC, Torres E, Hatzistergos KE, Hehre D, Suguihara C, Hare JM: Inhibition of the SDF-1/CXCR4 axis attenuates neonatal hypoxia-induced pulmonary hypertension. Circ Res 2009;104:1293-1301.

24 Ghosh MC, Makena PS, Gorantla V, Sinclair SE, Waters CM: CXCR4 regulates migration of lung alveolar epithelial cells through activation of Rac1 and matrix metalloproteinase-2. Am J Physiol Lung Cell Mol Physiol 2012; 302:L846-L856. 